\title{
Medidas de radón en espacios de trabajo subterráneos del Campus de la Universidad de Alicante
}

\author{
Radon measurements in underground workplaces \\ in the Campus of the University of Alicante
}

B. Piedecausa $^{*}$

\section{RESUMEN}

Las medidas del gas radiactivo radón en lugares de trabajo subterráneos del Campus de la Universidad de Alicante fueron realizadas mediante dispositivos E-PERM ${ }^{\circledR}$ de corto plazo durante el invierno de 2010 y el verano de 2011. Las concentraciones de radón obtenidas muestran valores máximos de $73,8 \mathrm{~Bq} / \mathrm{m}^{3}$ en invierno y $84,0 \mathrm{~Bq} / \mathrm{m}^{3}$ en verano, así como una media aritmética de $30,3 \mathrm{~Bq} / \mathrm{m}^{3}$.

Aunque no se abordan concentraciones medias anuales, todos los resultados se encuentran por debajo de los valores recogidos en la Recomendación 90/143/ EURATOM de la Comisión Europea relativa a la protección de la población contra los peligros de una exposición al radón en el interior de edificios así como los establecidos por la Instrucción IS-33 del Consejo de Seguridad Nuclear en lugares de trabajo, contribuyendo a la evaluación de la distribución de radón a nivel nacional y prestando atención a los problemas que este gas puede ocasionar en espacios subterráneos.

$319-7$

Palabras clave: Edificación subterránea; electret; radiación natural; radón interior.

\section{SUMMARY}

Measurements of radioactive radon gas in underground workplaces in the Campus of the University of Alicante were performed using short period E-PERM ${ }^{\circledast}$ devices during the winter of 2010 and summer of 2011. The obtained radon concentrations show maximum values of $73.8 \mathrm{~Bq} / \mathrm{m}^{3}$ in winter and $84.0 \mathrm{~Bq} / \mathrm{m}^{3}$ in summer, and an arithmetic mean of $30.3 \mathrm{~Bq} / \mathrm{m}^{3}$.

Despite not considering annual mean concentrations, results are below those included in 90/143/Euratom European Commission Recommendation on the protection of the public against the dangers of radon exposure inside buildings, as well as those established by the Nuclear Security Council IS-33 Instruction in workplaces, contributing to the evaluation of the national distribution of radon and paying attention to radon problems in underground spaces.

Keywords: Underground construction; electret; natural radiation; indoor radon.

\footnotetext{
(*) Universidad de Alicante (España). 


\section{INTRODUCCIÓN}

\subsection{El gas radón en los edificios}

El radón $\left({ }^{222} \mathrm{Rn}\right)$ es un gas noble radiactivo más pesado que el aire, inerte, incoloro, inodoro, insípido e invisible que presenta un período de semidesintegración de 3,8 días y que, en su proceso de desintegración desde los elementos inestables hasta el isótopo estable ${ }^{206} \mathrm{~Pb}$, produce descendientes sólidos con vidas medias inferiores a 30 minutos $\left({ }^{218} \mathrm{Po},{ }^{214} \mathrm{~Pb},{ }^{214} \mathrm{Bi}\right.$ y $\left.{ }^{214} \mathrm{Po}\right)$. Estos metales pesados, químicamente muy activos, pueden fijarse a partículas de aerosoles en el aire ambiental y, tras su inhalación, presentan una alta probabilidad de desintegrarse en nuestro sistema respiratorio, aumentando el riesgo de desarrollar enfermedades cancerígenas. Así, la Organización Mundial de la Salud (WHO) declara en 1986 el carácter cancerígeno del radón, confirmando su relación con el cáncer de pulmón tras diferentes estudios epidemiológicos mundiales (1) (2).

El interés por la problemática de la acumulación de radón en edificios se desarrolla mayoritariamente a finales de los años 70, cuando se convierte en una de las causas asociadas al Síndrome del Edificio Enfermo; un problema que, según la WHO, está ampliamente extendido en nuestras construcciones (un $30 \%$ de los edificios en los países industrializados resultan afectados por estas deficiencias) y que responde a un deterioro biológico de los materiales por hongos, ácaros, etc.; a un deterioro físico por ruidos, mala ventilación, etc. o a un deterioro químico por el $\mathrm{CO}_{2}$, radón, asbestos, etc.

Además de estos problemas, existen otros agravantes sociológicos ya que la permanencia del hombre urbano en ambientes cerrados donde pasa una media del $75 \%$ de su tiempo (Ilegando incluso hasta el 90\% en el caso de niños, ancianos o enfermos crónicos) ha provocado un creciente interés en el estudio de condiciones ambientales interiores con efectos nocivos sobre la salud, a fin de poder evitarlas.

A nivel global, existen recomendaciones y normativas tanto europeas como internacionales que instan a controlar la exposición a este gas en viviendas (mediante la intervención) y en lugares de trabajo (mediante la intervención y un control más regular) particularmente en espacios subterráneos (3) (4) (5) (6) (7) (8) (9) al ser lugares donde, al existir una mayor superficie en contacto directo con el terreno donde éste se genera, las concentraciones de radón pueden ser excepcionalmente altas.
En España, existe una mayor regulación respecto al control de la radiactividad ambiental en lugares de trabajo ya que, en 2001, se publica el Reglamento de Protección Sanitaria contra las Radiaciones Ionizantes (10), donde se recomienda realizar estudios en todos aquellos lugares de trabajo cuyo personal pueda estar expuesto a concentraciones elevadas de radón, torón y sus descendientes. Posteriormente, a finales de 2010, se publica el RD 1439/2010 (11) que modifica el anterior e introduce la necesidad de medir las concentraciones de radón en lugares de trabajo que puedan provocar un incremento significativo de la exposición de los trabajadores; y, en enero de 2012, el Consejo de Seguridad Nuclear (CSN) publica la Instrucción IS-33 (12), con el objetivo de establecer los criterios radiológicos para la protección frente a la radiactividad natural para las autoridades competentes y para los titulares de las actividades laborales afectadas.

\subsection{Antecedentes: estudios de radón en espacios subterráneos en España}

Debido a que las concentraciones de gas radón en espacios interiores se relacionan fundamentalmente con las condiciones del terreno donde se ubica la construcción, se considera muy pertinente el análisis de la influencia de este gas en aquellos espacios en contacto directo con el suelo o en aqueIlos situados en zonas más proclives a su depósito (conocidas como "radon prone areas"), recomendándose que las acciones emprendidas para disminuir estas concentraciones sean dirigidas prioritariamente hacia dichos ámbitos (13).

Con el fin de estudiar la distribución de este gas radiactivo en el territorio nacional, en los últimos años se han realizado estudios de concentraciones de radón en lugares de trabajo subterráneos de naturaleza muy diversa, como minas de uranio (14), cuevas turísticas (15) (16) o cuevas volcánicas (17); y más recientemente, la Universidad de Extremadura ha llevado a cabo un estudio de estas concentraciones en 130 lugares de trabajo distribuidos por toda la comunidad de Extremadura (18), a lo que hay que añadir la importancia de otras medidas en lugares de trabajo subterráneos de Cataluña tales como minas en explotación, antiguas minas con uso actual de museos, cuevas turísticas o aparcamientos subterráneos (19).

Por otra parte, con el fin de conocer la influencia de este gas en nuestras edificaciones, también se han estudiado concentraciones de radón en viviendas de distintos puntos de la geografía española (20) o particularizando en zonas concretas como 
en el caso de Madrid (21), Galicia (22) o Alicante (23), obteniéndose algunos resultados que sobrepasan los valores indicados por la Unión Europea en su Recomendación 90/143/Euratom (7) respecto a los niveles máximos aconsejables en espacios interiores, por lo que se hace necesario seguir realizando estudios experimentales a este respecto en nuestra geografía.

\section{OBJETO DEL ESTUDIO}

\subsection{Campus de la Universidad de Alicante}

En cuanto a la determinación de la existencia del gas radón en lugares de trabajo, el reciente Real Decreto 1439/2010 (11) indica que cualquier edificio con estancias subterráneas en contacto con el terreno ha de ser estudiado con el fin de descartar la existencia de radón que perjudique a sus trabajadores. Por tanto, este reglamento obliga a los gestores de actividades profesionales que implican tales fuentes a llevar a cabo estudios para determinar si hay un aumento significativo de la exposición de sus trabajadores o de los miembros del público que puedan no ser despreciables desde el punto de vista de la protección radiológica.

Así, tras los estudios anteriormente citados en lugares de trabajo en España y la publicación de otros trabajos con los mismos métodos y en entornos similares (24), se cree conveniente el análisis pormenorizado de la influencia del gas radón en un emplazamiento tan significativo como es la Universidad de Alicante: un lugar donde multitud de usuarios pasan la mayor parte de su tiempo de estudio o de trabajo durante el día y, por tanto, un lugar donde el tiempo de exposición a este gas puede llegar a ser bastante significativo.

El objetivo del presente trabajo es el análisis y la medida de la concentración de radón en el interior de espacios subterráneos de distintos edificios del Campus de la Universidad de Alicante, con el fin de determinar la concentración de radón existente en sus instalaciones, llegando a tomar las medidas correctivas al respecto en caso de considerarse necesario.

El citado Campus se encuentra a unos $5 \mathrm{~km}$ al noroeste de la ciudad de Alicante $\left(38^{\circ} 23^{\prime}\right.$ 4.7" N $0^{\circ} 30^{\prime} 47.33^{\prime \prime} \mathrm{O}$, ubicado a $105 \mathrm{~m}$ por encima del nivel del mar) y se extiende en un espacio de cerca de un millón de metros cuadrados (Figura 1). Un espacio que acoge actualmente alrededor de 3.700 miembros de personal académico, investigador y administrativo, además de 28.800 estudiantes de grado y postgrado.

\subsection{Selección de edificios estudiados}

Para la elección de las zonas de estudio en el Campus universitario se consideran en un primer momento los edificios con sótano excavado, al ser lugares con un contacto directo con el terreno, siendo susceptibles de una mayor influencia ante el radón. De este modo, aparece una primera lista de posibles emplazamientos, formada por 21 edificios (Tabla 1).

Tras el análisis de los usos globales en sótano, el nivel de ocupación de los espacios, las condiciones de ventilación, la distribución interior y la posible influencia del terreno natural, finalmente se decide estudiar de foma pormenorizada respecto al radón los 11 primeros edificios de la lista anterior y así evaluar el posible riesgo derivado de la exposición a este gas en distintos lugares de trabajo del Campus (Figura 2).

Tabla 1. Edificios del Campus de la Universidad de Alicante con estancias en sótano en contacto con el terreno.

\begin{tabular}{|l|l|}
\hline \multicolumn{2}{|c|}{ Edificios del campus con estancias en sótano } \\
\hline 1. Aulario General II & $\begin{array}{l}\text { 12. Escuela Universitaria De Óptica } \\
\text { y Optometría }\end{array}$ \\
\hline 2. Rectorado Y Servicios Generales & 13. Museo De La Universidad De Alicante \\
\hline $\begin{array}{l}\text { 3. Facultad De Económicas } \\
\text { y Empresariales }\end{array}$ & 14. Club Social II \\
\hline 4. Centro Comercial & 15. Torre De Control \\
\hline 5. Edificio "Germán Bernácer" & 16. Área De Experimentación Industrial \\
\hline 6. Biblioteca General & 17. Facultad De Ciencias I \\
\hline 7. Ciencias Sociales & 18. Facultad De Filosofía y Letras II \\
\hline 8. Escuela Politécnica Superior IV & 19. Aulario General I \\
\hline 9. Club Social III & 20. Petrología \\
\hline 10. Facultad De Ciencias VI & 21. Institutos Universitarios \\
\hline 11. Zona Deportiva & \\
\hline
\end{tabular}

Fuente: propia.
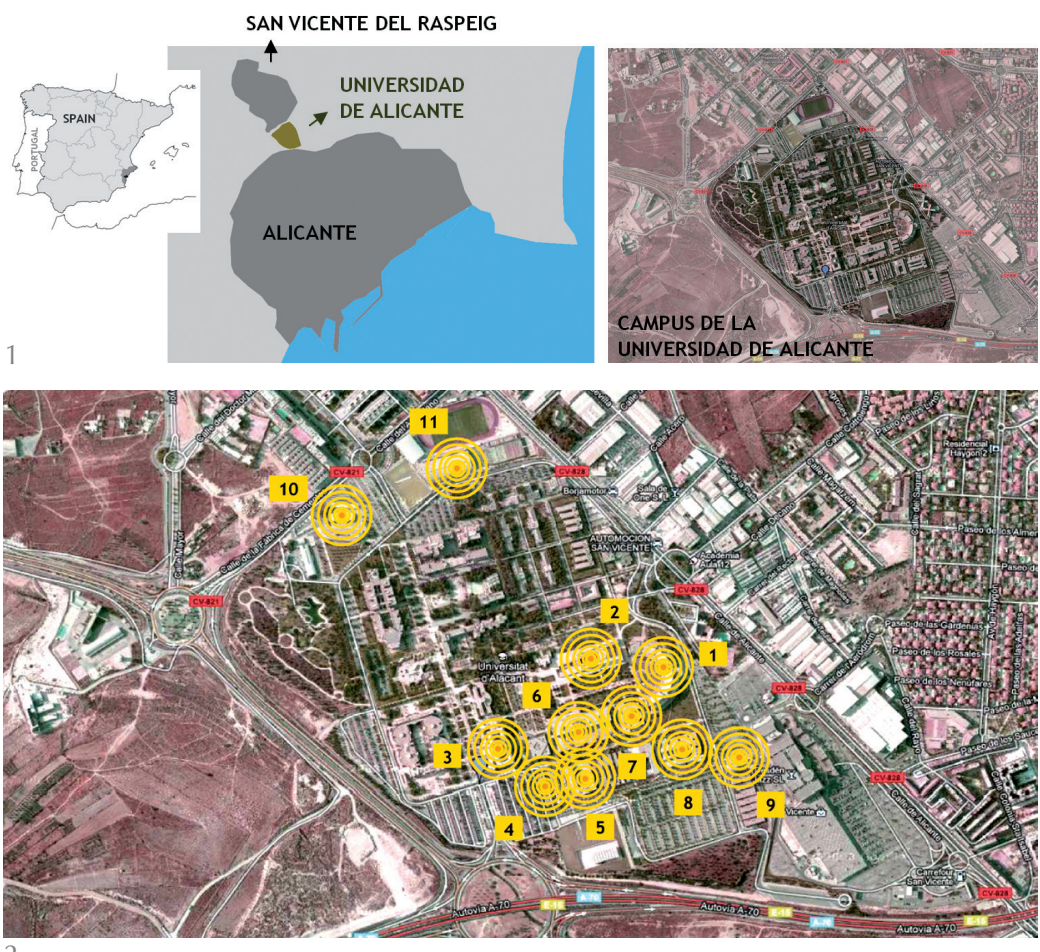


\section{MATERIALES Y MÉTODOS}

El presente trabajo se centra en el análisis de once edificios donde los usuarios pasan gran parte de su tiempo en el Campus, estudiando distintas estancias con uso de aulas comunes, zonas de informática, salones de actos y zonas administrativas ubicadas en nivel de sótano. Además, con el fin de obtener una visión más global del Campus, en algunos casos también se ha considerado interesante la medida en otros espacios de uso almacén o zonas de archivo, aunque en ningún caso baños o zonas de instalaciones, al ser espacios con ventilación forzada que puede alterar los resultados obtenidos.

Los edificios estudiados, de construcción reciente, están compuestos en su mayor parte por paramentos de ladrillo o bloque de hormigón, con muros de sótano y estructura de hormigón. Presentan sistemas centralizados de calefacción en invierno y aire acondicionado en verano en grandes espacios como aulas, zonas de servicios o zonas comunes; aunque existen zonas de despachos o pequeños espacios que se acondicionan mediante sistemas individualizados.

Las mediciones de radón del presente estudio se han realizado con dispositivos E-PERM (electret) de tipo " $\mathrm{S}$ " disponibles en el mercado (Rad Elec Inc., Frederick, MD 21701, EE.UU.). El sistema utiliza dispositivos pasivos integradores reutilizables que se emplazan en el lugar de medición durante un período de tiempo y que, posteriormente, se introducen en un lector de voltaje que determina la concentración de radón en dicho espacio (25) (26).

En todos los lugares de trabajo estudiados, los detectores estuvieron expuestos de 5 a 7 días durante el invierno de 2010 y el verano de 2011; y en algunos casos fueron instalados pares de detectores para estimar la fiabilidad de la concentración obtenida. Durante las medidas, se ha recuperado el $100 \%$ de los dispositivos utilizados y no han existido incidencias a considerar en los distintos periodos de exposición. Además, aparte de la disposición de detectores de radón, en todos los espacios estudiados se midieron los niveles de radiación gamma ambiental mediante un dispositivo portátil de tipo Dosimeter PM 1203M, con el fin de establecer experimentalmente el fondo existente.

Acabada la exposición y una vez recogidos los detectores, los datos obtenidos de los electretes se relacionan mediante una aplicación informática con un factor de calibración y con el tiempo de duración de las medidas, obteniéndose los valores de las concentraciones de radón ambiental en $\mathrm{Bq} / \mathrm{m}^{3}$ existentes en cada estancia. En este estudio no se ha considerado la corrección de fondo ambiental debido a que nos encontramos por debajo de los $1.200 \mathrm{~m}$ respecto al nivel del mar, el mínimo valor a partir del que ha de considerarse.

\subsection{Selección de detectores y época de medida}

Con el objeto de establecer un primer rango de magnitud en la valoración de las concentraciones de radón en distintos espacios de trabajo subterráneos, el presente estudio plantea tiempos cortos de medida en cada periodo estacional.

En la campaña se han empleado cámaras y electretes de corto plazo (SST) en estancias con puertas y ventanas exteriores cerradas (sin ventilación alguna), que se encuentran desocupadas durante el periodo de medida aprovechando fines de semana o días de vacaciones. De este modo, se ha pretendido analizar el caso más desfavorable en cada espacio respecto a la máxima concentración posible de radón, aún asumiendo que puedan existir desviaciones con respecto a la media anual si las estancias fueran analizadas durante un periodo de tiempo mayor y en unas condiciones normales de ventilación.

Para la elección de la época más idónea de medida, se considera pertinente un análisis de cada espacio en dos periodos estacionales distintos, con idéntico tiempo de exposición. Así, se realizan medidas en las condiciones más desfavorables con dispositivos SST en época de invierno (diciembre) y verano (junio) con el fin de comprobar si existen diferencias significativas entre ambos resultados. Con estas premisas, se establece el número de medidas y el tiempo de exposición para cada edificio recogidos en la campaña de la Tabla 2.

\subsection{Fichas descriptivas de edificios}

Con el fin de representar las condiciones propias de cada espacio analizado, se consideró necesaria la definición de fichas descriptivas para los edificios estudiados (Figura 3), compuestas por un apartado de datos generales (localización en el edificio, horas de ocupación habituales, superficie de la estancia, posibilidad de ventilación al exterior, elementos en contacto con el terreno), una descripción funcional (diferenciación de estancias), distintas imágenes interiores y exteriores, así como planos de distribución de las estancias. 
Además, estas fichas también recogen datos particulares sobre el estudio del radón ambiental en dicha estancia, con un apartado sobre el muestreo realizado (número de detectores y ubicación) y una descripción de las condiciones de medida (tiempo de exposición, estación del año, condiciones ambientales, temperatura interior/exterior en inicio y final) (Figura 3).
3. Ficha tipológica de identificación de cada edificio del Campus analizado.

Fuente: propia.

Tabla 2. Principales características de las medidas de radón realizadas en la campaña.

\begin{tabular}{|c|c|c|c|c|c|}
\hline & Edificio & Ubicación & Fechas & Tiempo & $\begin{array}{l}\text { Núm. De } \\
\text { Medidas }\end{array}$ \\
\hline 01 & Aulario II & Zona de reprografía & $\begin{array}{l}\text { Invierno-Dic } \\
\text { Verano-Jun }\end{array}$ & $\begin{array}{l}6 \text { días } \\
5 \text { días }\end{array}$ & $\begin{array}{l}2 \mathrm{SST} \\
1 \mathrm{SST}\end{array}$ \\
\hline 02 & Rectorado & Zona de archivo y almacén & $\begin{array}{l}\text { Invierno-Dic } \\
\text { Verano-Jun }\end{array}$ & $\begin{array}{l}6 \text { días } \\
7 \text { días }\end{array}$ & $\begin{array}{l}2 \text { SST } \\
4 \text { SST }\end{array}$ \\
\hline 03 & Económicas y Empresariales & Aulas de informática & $\begin{array}{l}\text { Invierno-Dic } \\
\text { Verano-Jun }\end{array}$ & $\begin{array}{l}6 \text { días } \\
5 \text { días }\end{array}$ & $\begin{array}{l}2 \mathrm{SST} \\
2 \mathrm{SST}\end{array}$ \\
\hline 04 & Centro Comercial & $\begin{array}{l}\text { Almacén del Museo de la } \\
\text { Universidad de Alicante }\end{array}$ & $\begin{array}{l}\text { Invierno-Dic } \\
\text { Verano-Jun }\end{array}$ & $\begin{array}{l}6 \text { días } \\
7 \text { días }\end{array}$ & $\begin{array}{l}2 \text { SST } \\
2 \text { SST }\end{array}$ \\
\hline 05 & Edificio "Germán Bernácer" & Salón de actos & $\begin{array}{l}\text { Invierno-Dic } \\
\text { Verano-Jun }\end{array}$ & $\begin{array}{l}6 \text { días } \\
5 \text { días }\end{array}$ & $\begin{array}{l}2 \mathrm{SST} \\
1 \mathrm{SST}\end{array}$ \\
\hline 06 & Biblioteca General & Zona de admin. $y$ archivo & $\begin{array}{l}\text { Invierno-Dic } \\
\text { Verano-Jun }\end{array}$ & $\begin{array}{l}6 \text { días } \\
7 \text { días }\end{array}$ & $\begin{array}{l}2 \text { SST } \\
3 \text { SST }\end{array}$ \\
\hline 07 & Ciencias Sociales & Aula de teoría & $\begin{array}{l}\text { Invierno-Dic } \\
\text { Verano-Jun }\end{array}$ & $\begin{array}{l}6 \text { días } \\
5 \text { días }\end{array}$ & $\begin{array}{l}2 \text { SST } \\
1 \text { SST }\end{array}$ \\
\hline 08 & Escuela Polit. Superior IV & Salón de actos y aula teoría & $\begin{array}{l}\text { Invierno-Dic } \\
\text { Verano-Jun }\end{array}$ & $\begin{array}{l}6 \text { días } \\
5 \text { días }\end{array}$ & $\begin{array}{l}2 \text { SST } \\
2 \text { SST }\end{array}$ \\
\hline 09 & Club Social III & Zona de almacén común & $\begin{array}{l}\text { Invierno-Dic } \\
\text { Verano-Jun }\end{array}$ & $\begin{array}{l}6 \text { días } \\
7 \text { días }\end{array}$ & $\begin{array}{l}2 \text { SST } \\
2 \text { SST }\end{array}$ \\
\hline 10 & Facultad de Ciencias VI & Aulas de teoría & $\begin{array}{l}\text { Invierno-Dic } \\
\text { Verano-Jun }\end{array}$ & $\begin{array}{l}6 \text { días } \\
5 \text { días }\end{array}$ & $\begin{array}{l}4 \text { SST } \\
4 \text { SST }\end{array}$ \\
\hline 11 & Zona Deportiva & Zona de personal de servicios & $\begin{array}{l}\text { Invierno-Dic } \\
\text { Verano-Jun }\end{array}$ & $\begin{array}{l}6 \text { días } \\
7 \text { días }\end{array}$ & $\begin{array}{l}1 \mathrm{SST} \\
1 \mathrm{SST}\end{array}$ \\
\hline
\end{tabular}

Fuente: propia.

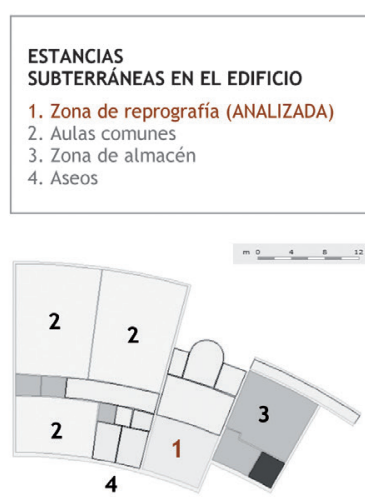

Plano de estancias existentes en la planta sótano del Fuente: propia a partir del Servicio de Cartografia de Fuente: propia a partiri
Universidad de Alicante.

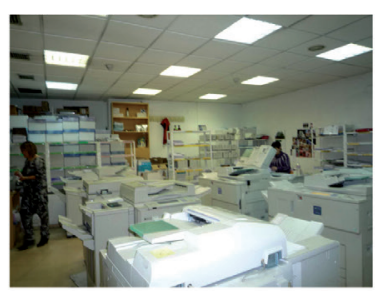

\begin{tabular}{l}
\hline edificio 1. Aulario II \\
\hline ESPACIO \\
ZONA DE REPROGRAFÍA \\
\hline
\end{tabular}

1. DATOS GENERALES

Espacio analizado: zona de reprografía Ocupación de usuarios: $9 \mathrm{~h}$ diarias

- Superficie de la estancia: $51,5 \mathrm{~m}^{2}$

Ventilación al exterior: No, accede a un pasillo interior

Contacto con el terreno: suelo y dos paredes

\section{MUESTREO REALIZADO}

Número de detectores: 2 en dic. 2010 y 1 en jun. 2011

Ubicación de los detectores: encima de una estantería

2.1. MEDIDAS EN DICIEMBRE 2010

Estación del año: Invierno

Fecha inicio: 23 de diciembre de 2010

Fecha final: 29 de diciembre de 2011

Tiempo total de exposición: 6 días

Condiciones: espacio cerrado durante las medidas

2.2. MEDIDAS EN JUNIO DE 2011

Estación del año: Verano

Fecha inicio: 22 de junio de 2011

Fecha final: 27 de junio de 2011

Tiempo total de exposición: 5 días

Condiciones: espacio cerrado durante las medidas

\section{CONDICIONES AMBIENTALES DURANTE LA MEDIDA}

\begin{tabular}{|l|c|c|l|c|c|}
\hline Datos & $\begin{array}{c}\text { Dic } \\
2010\end{array}$ & $\begin{array}{c}\text { Jun } \\
2011\end{array}$ & Datos & $\begin{array}{c}\text { Dic } \\
\mathbf{2 0 1 0}\end{array}$ & $\begin{array}{c}\text { Jun } \\
\mathbf{2 0 1 1}\end{array}$ \\
\hline Temperatura interior & $26,8^{\circ} \mathrm{C}$ & $25,8{ }^{\circ} \mathrm{C}$ & Temperatura exterior & $18^{\circ} \mathrm{C}$ & $29,4{ }^{\circ} \mathrm{C}$ \\
\hline Humedad relat. interior & $51,3 \%$ & $48,9 \%$ & Humedad relat.exterior & $55,2 \%$ & $75,2 \%$ \\
\hline
\end{tabular}


4. Fichas de los resultados de radón ambiental obtenidos en cada edificio analizado. Fuente: propia.

\section{RESULTADOS}

Con el fin de representar los resultados, se decide documentar los valores obtenidos en otra serie de fichas de radón ambiental para cada uno de los edificios analizados (Figura 4), compuestas por imágenes y planos de ubicación de los detectores utilizados en la estancia, los datos de la radiación gamma ambiental obtenida así como tablas de los resultados tanto en invierno como en verano (fechas de inicio/fin de la exposición, concentración de radón obtenida e incertidumbre de las medidas).

A modo de resumen, a continuación se adjunta una tabla con todos los resultados obtenidos durante las medidas de invierno y verano en cada uno de los edificios del Campus estudiados en la campaña (Tabla 3).

Así, en las medidas durante la época de invierno, se obtiene que la mayor concentración registrada corresponde a un valor de $77,0 \mathrm{~Bq} / \mathrm{m}^{3}$ en un espacio de almacén del Centro Comercial (edificio 4), mientras que el valor más bajo corresponde a 5,6 Bq/ $\mathrm{m}^{3}$ en un espacio de almacén en el Club Social III (edificio 9).

Por otra parte, en las medidas durante la época de verano, se obtiene que la mayor concentración registrada corresponde a un valor de $89,0 \mathrm{~Bq} / \mathrm{m}^{3}$ en el mismo espacio de almacén del Centro Comercial (edificio 4), mientras que el valor más bajo corresponde a un valor de 5,7 Bq/ $\mathrm{m}^{3}$ en el mismo espacio de almacén en el Club Social III (edificio 9). Cabe destacar que los valores de presión atmosférica se mantuvieron en un rango constante durante los distintos periodos de medida y no se registró precipitación alguna; por ello se han considerado las condiciones atmosféricas exteriores como estables.

A nivel global, teniendo en cuenta las medidas realizadas en ambos periodos, se establece que las concentraciones de actividad del gas radón interior en los sótanos del Campus de la Universidad de Alicante oscilan entre $7,2 \mathrm{~Bq} / \mathrm{m}^{3}$ y $73,8 \mathrm{~Bq} / \mathrm{m}^{3}$ en la época de invierno y entre $6,4 \mathrm{~Bq} / \mathrm{m}^{3}$ y $84,0 \mathrm{~Bq} / \mathrm{m}^{3}$ en la época de verano (Tabla 4); con unas concentraciones medias totales entre 6,8 y $78,9 \mathrm{~Bq} / \mathrm{m}^{3}$ (media aritmética).

Si se establece una comparativa gráfica entre los resultados obtenidos en las medidas en época del invierno de 2010 frente a las del verano 2011, se puede determinar que no existe una variación estacional estadísticamente significativa en la presente campaña (Figura 5). Sin embargo, estos resultados no pueden ser generalizados a otros casos similares ya que, según han demostrado distintos estudios internacionales, la concentración interior de radón puede ser muy variable de un edificio a otro e incluso dentro de un mismo edificio.

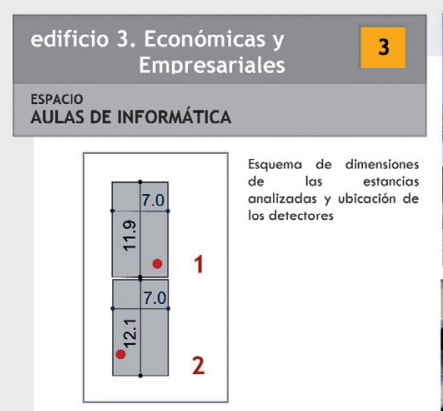

4. RESULTADOS OBTENIDOS

- Radiación gamma ambiental de fondo: $0,07 \mu \mathrm{Sv} / \mathrm{h}$

Los resultados de radón ambiental obtenidos con el muestreo realizado mediante dispositivos E-PERM se detallan en las tablas siguientes:

\begin{tabular}{|c|c|c|c|c|c|c|}
\hline \multicolumn{7}{|c|}{ MEDIDAS EN DICIEMBRE 2010} \\
\hline Espacio & Inicio & Fin & $\begin{array}{l}N^{\circ} \text { de } \\
\text { días }\end{array}$ & $\begin{array}{c}\text { Concent. } \\
\text { Radón } \\
\mathrm{Bq} / \mathrm{m}^{3}\end{array}$ & $\begin{array}{l}\text { Incertid. } \\
(+/-) \mathrm{Bq} / \mathrm{m}^{3}\end{array}$ & $\begin{array}{l}\text { Incertid. } \\
(+/-) \text { \%Error }\end{array}$ \\
\hline $\begin{array}{l}\text { 1.Aula } \\
\text { informát. }\end{array}$ & $\begin{array}{c}29 / 12 / 10 \\
13: 15\end{array}$ & $\begin{array}{c}04 / 01 / 111 \\
09: 45\end{array}$ & 6 & 24,3 & 2 & 10 \\
\hline $\begin{array}{l}\text { 2.Aula } \\
\text { informát. }\end{array}$ & $\begin{array}{c}29 / 12 / 10 \\
13: 20\end{array}$ & $\begin{array}{c}04 / 01 / 11 \\
09: 45\end{array}$ & 6 & 28,5 & 4 & 8 \\
\hline \multicolumn{7}{|c|}{ MEDIDAS EN JUNIO DE 2011} \\
\hline Espacio & Inicio & Fin & $\begin{array}{l}N^{\circ} \text { de } \\
\text { días }\end{array}$ & $\begin{array}{l}\text { Concent. } \\
\text { Radón } \\
\mathrm{Bq} / \mathrm{m}^{3}\end{array}$ & $\begin{array}{l}\text { Incertid. } \\
(+/-)) \mathrm{Bq} / \mathrm{m}^{3}\end{array}$ & $\begin{array}{l}\text { Incertid. } \\
(+/-) \text { \%Error }\end{array}$ \\
\hline $\begin{array}{l}\text { 1. Aula } \\
\text { informat. }\end{array}$ & $\begin{array}{c}22 / 06 / 11 \\
07: 55\end{array}$ & $\begin{array}{c}27 / 06 / 11 \\
08: 00\end{array}$ & 5 & 53,4 & 4 & 8 \\
\hline $\begin{array}{l}\text { 2.Aula } \\
\text { informat. }\end{array}$ & $\begin{array}{c}22 / 06 / 11 \\
08: 00\end{array}$ & $\begin{array}{c}27 / 06 / 11 \\
08: 02\end{array}$ & 5 & 49,8 & 4 & 8 \\
\hline
\end{tabular}

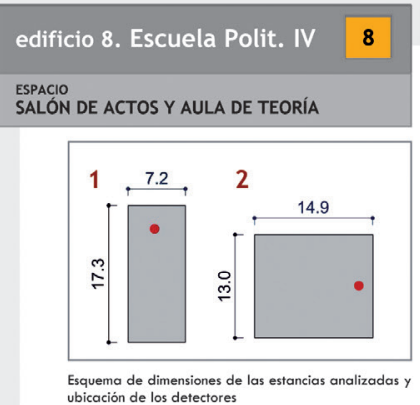

\section{RESULTADOS OBTENIDOS}

Radiación gamma ambiental de fondo: $0,07 \mu 5 \mathrm{v} / \mathrm{h}$

Los resultados de radón ambiental obtenidos con el muestreo realizado mediante dispositivos E-PERM se detallan en las tablas siguientes:

\begin{tabular}{|c|c|c|c|c|c|c|}
\hline \multicolumn{7}{|c|}{ MEDIDAS EN DICIEMBRE 2010} \\
\hline Espacio & Inicio & Fin & $\begin{array}{l}\mathrm{N}^{0} \text { de } \\
\text { días }\end{array}$ & $\begin{array}{c}\text { Concent. } \\
\text { Radón } \\
\text { Bq/m } \mathrm{m}^{3}\end{array}$ & $\begin{array}{l}\text { Incertid. } \\
(+1-) \mathrm{Bq} / \mathrm{m}^{3}\end{array}$ & $\begin{array}{l}\text { Incertid. } \\
(+/-) \text { \%Error }\end{array}$ \\
\hline 1.S.Actos & $\begin{array}{c}29 / 12 / 10 \\
12: 07\end{array}$ & $\begin{array}{c}04 / 01 / 11 \\
09: 02\end{array}$ & 6 & 26,9 & 2 & 9 \\
\hline 2.Aula & $\begin{array}{c}29 / 12 / 10 \\
12: 11\end{array}$ & $\begin{array}{c}04 / 01 / 11 \\
09: 03\end{array}$ & 6 & 23,9 & 2 & 10 \\
\hline \multicolumn{7}{|c|}{ MEDIDAS EN JUNIO DE 2011} \\
\hline Espacio & Inicio & Fin & $\begin{array}{l}\mathrm{N}^{0} \text { de } \\
\text { dias }\end{array}$ & $\begin{array}{c}\text { Concent. } \\
\text { Radón } \\
\mathrm{Bq} / \mathrm{m}^{3}\end{array}$ & $\begin{array}{l}\text { Incertid. } \\
(+/-) \mathrm{Bq} / \mathrm{m}^{3}\end{array}$ & $\begin{array}{l}\text { Incertid. } \\
(+/-) \text { \%Error }\end{array}$ \\
\hline 1.S.Actos & $\begin{array}{c}22 / 06 / 11 \\
09: 38\end{array}$ & $\begin{array}{c}27 / 06 / 11 \\
08: 40\end{array}$ & 5 & 40,5 & 4 & 9 \\
\hline 2.Aula & $\begin{array}{c}22 / 06 / 11 \\
09: 30\end{array}$ & $\begin{array}{c}27 / 06 / 11 \\
08: 45\end{array}$ & 5 & 25,7 & 3 & 11 \\
\hline
\end{tabular}


Tabla 3. Tabla de los resultados de las medidas de radón en el interior de las estancias analizadas.

\begin{tabular}{|c|c|c|c|c|c|c|}
\hline Periodo & Ubicación & Uso & Inicio & Final & Días & $\begin{array}{l}\text { Concent. De } \\
\text { Radón bq/m }\end{array}$ \\
\hline Invierno & 1. Aulario II & Reprografía & 23/12/10 12:40 & 29/12/10 12:38 & 6,00 & 22,7 \\
\hline Invierno & 1. Aulario II & Reprografía & 23/12/10 12:40 & 29/12/10 12:38 & 6,00 & 25,7 \\
\hline Verano & 1. Aulario II & Reprografía & 22/06/11 09:10 & 27/06/11 10:18 & 5,05 & 43,4 \\
\hline Invierno & 2. Rectorado & Archivo & 23/12/10 12:18 & 29/12/10 10:30 & 5,93 & 23,2 \\
\hline Verano & 2. Rectorado & Archivo & 27/06/11 10:10 & 04/07/11 08:05 & 6,91 & 26,0 \\
\hline Verano & 2. Rectorado & Archivo & 27/06/11 10:10 & 04/07/11 08:05 & 6,91 & 23,8 \\
\hline Invierno & 2. Rectorado & Bajo salón actos & 23/12/10 12:22 & 29/12/10 10:31 & 5,92 & 53,0 \\
\hline Verano & 2. Rectorado & Bajo salón actos & 27/06/11 10:15 & 04/07/11 08:07 & 6,91 & 39,3 \\
\hline Verano & 2. Rectorado & Bajo salón actos & 27/06/11 10:15 & 04/07/11 08:07 & 6,91 & 36,7 \\
\hline Invierno & 3. Económicas & Informática 2 & 29/12/10 13:15 & 04/01/11 09:45 & 5,85 & 24,3 \\
\hline Verano & 3. Económicas & Informática 2 & 22/06/11 07:55 & 27/06/11 08:00 & 5,00 & 53,4 \\
\hline Invierno & 3. Económicas & Informática 1 & 29/12/10 13:20 & 04/01/11 09:45 & 5,85 & 28,5 \\
\hline Verano & 3. Económicas & Informática 1 & 22/06/11 08:00 & 27/06/11 08:02 & 5,00 & 49,8 \\
\hline Invierno & 4. Centro comercial & Almacén & 04/01/11 11:36 & 10/01/11 10:28 & 5,95 & 77,0 \\
\hline Invierno & 4. Centro comercial & Almacén & 04/01/11 11:36 & 10/01/11 10:28 & 5,95 & 70,5 \\
\hline Verano & 4. Centro comercial & Almacén & 27/06/11 09:46 & 04/07/11 08:45 & 6,96 & 78,9 \\
\hline Verano & 4. Centro comercial & Almacén & 27/06/11 09:46 & 04/07/11 08:45 & 6,96 & 89,0 \\
\hline Invierno & 5. Germán bernácer & Salón de actos & 29/12/10 13:35 & 04/01/11 09:20 & 5,82 & 39,2 \\
\hline Invierno & 5. Germán bernácer & Salón de actos & 29/12/10 13:35 & 04/01/11 09:19 & 5,82 & 39,7 \\
\hline Verano & 5. Germán bernácer & Salón de actos & 22/06/11 08:12 & 27/06/11 08:15 & 5,00 & 35,6 \\
\hline Invierno & 6. Biblioteca gral. & Depósito & 23/12/10 12:55 & 29/12/10 10:35 & 5,90 & 26,4 \\
\hline Verano & 6. Biblioteca gral. & Depósito & 27/06/11 10:00 & 04/07/11 08:55 & 6,95 & 76,8 \\
\hline Invierno & 6. Biblioteca gral. & Archivo & 23/12/10 13:05 & 29/12/10 10:38 & 5,90 & 23,4 \\
\hline Verano & 6. Biblioteca gral. & Archivo & 27/06/11 10:05 & 04/07/11 08:58 & 6,95 & 25,9 \\
\hline Verano & 6. Biblioteca gral. & Archivo & 27/06/11 10:05 & 04/07/11 08:58 & 6,95 & 33,6 \\
\hline Invierno & 7. Ciencias sociales & Aula 0034PS098 & 29/12/10 13:00 & 04/01/11 09:12 & 5,84 & 17,9 \\
\hline Invierno & 7. Ciencias sociales & Aula 0034PS098 & 29/12/10 13:00 & 04/01/11 09:12 & 5,84 & 21,3 \\
\hline Verano & 7. Ciencias sociales & Aula 0034PS100 & 22/06/11 08:30 & 27/06/11 08:21 & 4,99 & 7,9 \\
\hline Invierno & 8. Esc. Polit. IV & Salón de actos & 29/12/10 12:07 & 04/01/11 09:02 & 5,87 & 26,9 \\
\hline Invierno & 8. Esc. Polit. IV & Aula teoría & 29/12/10 12:11 & 04/01/11 09:03 & 5,87 & 23,9 \\
\hline Verano & 8. Esc. Polit. IV & Salón de actos & 22/06/11 09:38 & 27/06/11 08:40 & 4,96 & 40,5 \\
\hline Verano & 8. Esc. Polit. IV & Aula teoría & 22/06/11 09:30 & 27/06/11 08:45 & 4,97 & 25,7 \\
\hline Invierno & 9. Club social III & Almacén & 04/01/11 11:25 & 10/01/11 10:16 & 5,95 & 8,7 \\
\hline Invierno & 9. Club social III & Almacén & 04/01/11 11:25 & 10/01/11 10:16 & 5,95 & 5,6 \\
\hline Verano & 9. Club social III & Almacén & 27/06/11 09:35 & 04/07/11 08:30 & 6,95 & 5,7 \\
\hline Verano & 9. Club social III & Almacén & 27/06/11 09:35 & 04/07/11 08:30 & 6,95 & 7,0 \\
\hline Invierno & 10. Ciencias VI & Aula S002 & 29/12/10 14:05 & 04/01/11 09:34 & 5,81 & 9,4 \\
\hline Invierno & 10. Ciencias VI & Aula S002 & 29/12/10 14:05 & 04/01/11 09:34 & 5,81 & 12,3 \\
\hline Verano & 10. Ciencias VI & Aula S002 & $22 / 06 / 1108: 50$ & 27/06/11 07:38 & 4,95 & 11,4 \\
\hline Verano & 10. Ciencias VI & Aula S002 & 22/06/11 09:00 & 27/06/11 07:38 & 4,94 & 15,1 \\
\hline Invierno & 10. Ciencias VI & Aula S004 & 29/12/10 14:08 & 04/01/11 09:36 & 5,81 & 9,4 \\
\hline Invierno & 10. Ciencias VI & Aula S004 & 29/12/10 14:08 & 04/01/11 09:36 & 5,81 & 9,2 \\
\hline Verano & 10. Ciencias VI & Aula S004 & 22/06/11 09:00 & 27/06/11 07:45 & 4,95 & 8,4 \\
\hline Verano & 10. Ciencias VI & Aula S004 & 22/06/11 09:00 & 27/06/11 07:45 & 4,95 & 8,8 \\
\hline Invierno & 11. Zona deportiva II & Sala personal & 23/12/10 14:15 & 29/12/10 10:55 & 5,86 & 8,7 \\
\hline Verano & 11. Zona deportiva II & Sala personal & 27/06/11 08:15 & 04/07/11 08:20 & 6,91 & 9,2 \\
\hline
\end{tabular}

Fuente: propia. 
5. Resultados medios de las medidas de radón en el interior de los edificios analizados, en $\mathrm{Bq} / \mathrm{m}^{3}$.

Fuente: propia.

Tabla 4. Resultados medios de las medidas de radón en el interior de los edificios analizados, en $\mathrm{Bq} / \mathrm{m}^{3}$. En el caso de la existencia de dos medidas en un mismo espacio, primero se ha obtenido la media aritmética de los valores en dicho espacio para promediar posteriormente con otras estancias del edificio, obteniendo una concentración única para cada edificio estudiado.

\begin{tabular}{|c|c|c|c|c|}
\cline { 2 - 4 } \multicolumn{1}{c|}{} & Edificio & $\begin{array}{c}\text { Concentración } \\
\text { Media invierno } \\
\left(\mathbf{B q} / \mathbf{m}^{3}\right)\end{array}$ & $\begin{array}{c}\text { Concentración } \\
\text { Media verano } \\
\left(\mathbf{B q} / \mathbf{m}^{3}\right)\end{array}$ & $\begin{array}{c}\text { Concentración } \\
\text { Media total } \\
\left(\mathbf{B q} / \mathbf{m}^{3}\right)\end{array}$ \\
\hline $\mathbf{0 1}$ & Aulario II & 24,2 & 43,4 & 33,8 \\
\hline $\mathbf{0 2}$ & Rectorado & 38,1 & 31,5 & 34,8 \\
\hline $\mathbf{0 3}$ & Económicas y Empresariales & $\mathbf{2 6 , 4}$ & 51,6 & 39,0 \\
\hline $\mathbf{0 4}$ & Centro Comercial & $\mathbf{7 3 , 8}$ & 84,0 & 78,9 \\
\hline $\mathbf{0 5}$ & Edificio “Germán Bernácer" & 39,5 & 35,6 & 37,6 \\
\hline $\mathbf{0 6}$ & Biblioteca General & 24,9 & 53,3 & 39,1 \\
\hline $\mathbf{0 7}$ & Ciencias Sociales & 19,6 & 7,9 & 29,3 \\
\hline $\mathbf{0 8}$ & Escuela Politécnica Superior IV & 25,4 & 33,1 & 6,8 \\
\hline $\mathbf{0 9}$ & Club Social III & 7,2 & 6,4 & 11,7 \\
\hline $\mathbf{1 0}$ & Facultad de Ciencias VI & 10,1 & 13,25 & 9,0 \\
\hline $\mathbf{1 1}$ & Zona Deportiva & 8,7 & 9,2 & \\
\hline
\end{tabular}

Fuente: propia.

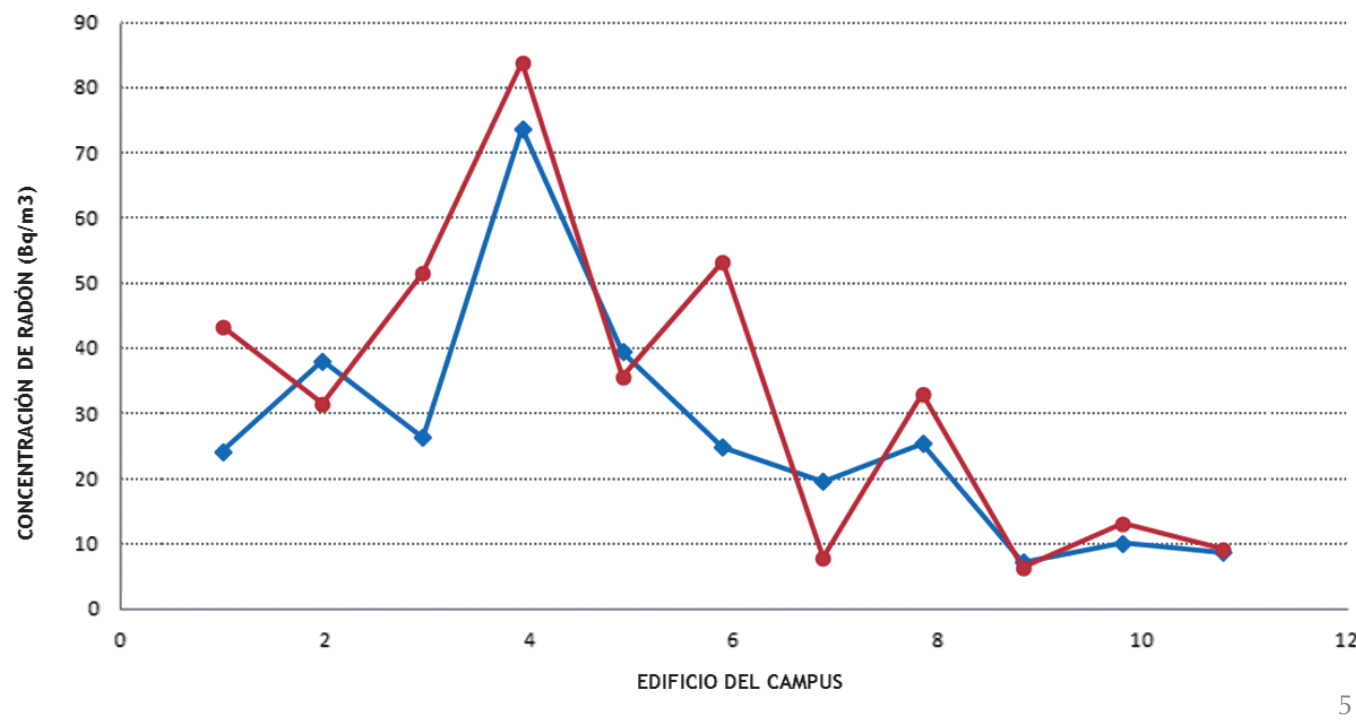

\section{CONCLUSIONES}

Aunque el radón siempre ha estado presente en el ambiente que respiramos, su concentración en nuestros espacios interiores ha aumentado significativamente en los últimos años debido a la existencia de una construcción cada vez más hermética, con mejor aislamiento térmico, menor ventilación y mayor ahorro energético. Así, nuestras edificaciones pueden alcanzar niveles de concentración de radón peligrosos preferentemente en lugares con baja ventilación como son sótanos, semisótanos, garajes u otras áreas en contacto directo con terreno, al ser éste su principal fuente emisora en la naturaleza y he aquí el interés del presente estudio.

De los resultados obtenidos respecto a la concentración de este gas en espacios subterráneos administrativos y docentes del Campus de la Universidad del Alicante se desprende que, de los edificios analizados, un $36,4 \%$ (4 edificios) se encuentra entre $0-20 \mathrm{~Bq} / \mathrm{m}^{3}$, un $54,6 \%$ (6 edificios) se encuentra entre $21-60 \mathrm{~Bq} / \mathrm{m}^{3}$ y un $9 \%$ (1 edificio) se encuentra por encima de $61 \mathrm{~Bq} / \mathrm{m}^{3}$, tal y como se muestra en el siguiente gráfico de intensidad (Figura 6).

El presente trabajo se ha planteado como un primer estudio del rango de la concentración de radón existente en distintos espacios de trabajo subterráneos de la universidad, sirviendo como base para el desarrollo posterior de otras campañas experimentales de mayor duración (en periodos de 3 a 6 meses) que permitan establecer valores promedio anuales para una caracterización más exhaustiva (medidas integradas/medidas en continuo), como la requerida por la Instrucción IS-33 (12) respecto a la exposición a radón en lugares de trabajo. 


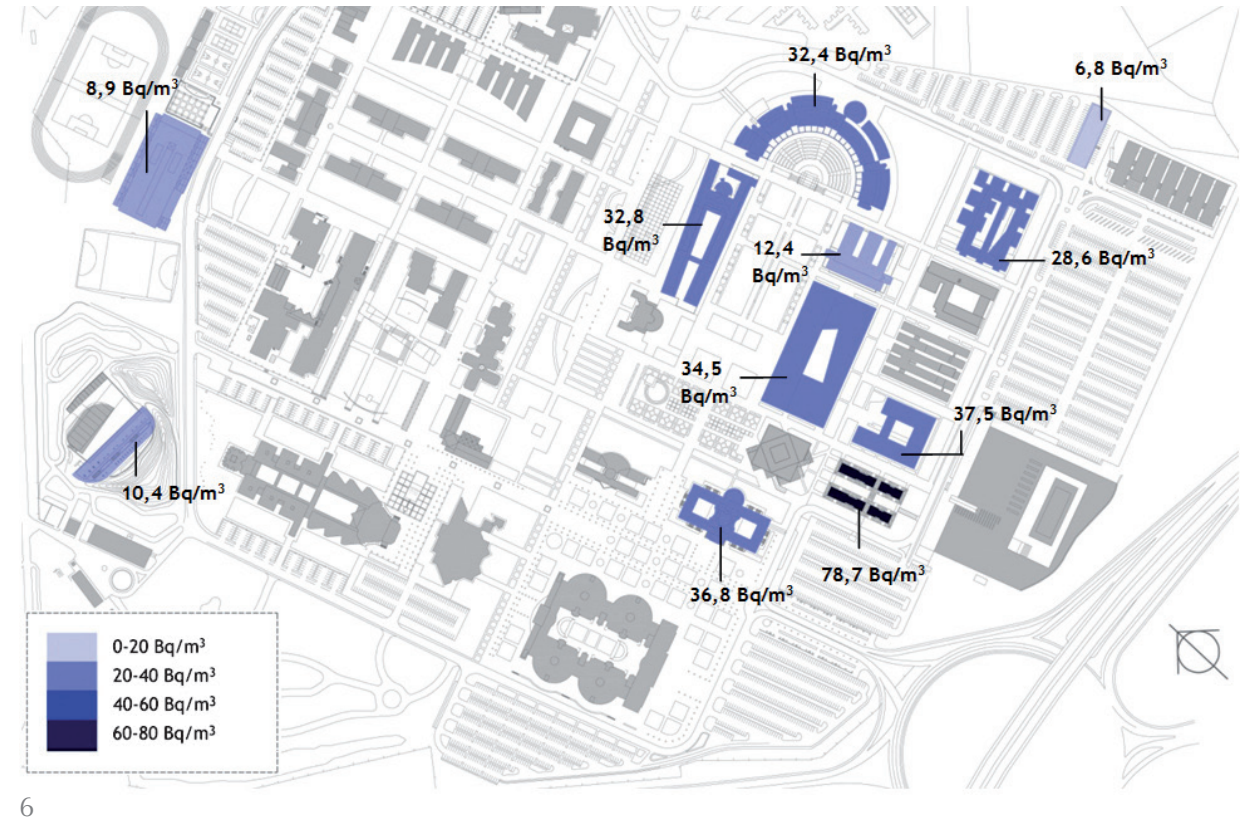

Teniendo en cuenta estas premisas, y aún considerando la necesidad de continuar la campaña iniciada con el fin de obtener la concentración de radón media anual para estos edificios, la media aritmética de la concentración de radón de las 46 medidas realizadas es de $30,3 \mathrm{~Bq} / \mathrm{m}^{3}$, un valor muy por debajo de los $300 \mathrm{~Bq} / \mathrm{m}^{3}$ de concentración de radón media anual estipulados por la IS-33 (12) para la adopción de medidas correctivas en lugares de trabajo con elevada permanencia de miembros del público.

Por otro lado, cabe destacar que a pesar de haberse analizado edificios construidos en años distintos y con distribuciones diferentes, todos los resultados obtenidos tanto en diciembre de 2010 como en junio de 2011 en los distintos edificios del campus son valores muy próximos entre sí al ser espacios con características similares y cercanos en ubicación; observándose, además, una escasa variación de los niveles medios de concentración de radón en función de la época del año.

Por ello, si se considera que tanto las estructuras, cimentaciones o tabiques de los edificios estudiados favorecen a una cierta estabilidad de la concentración interior a lo largo del año, en el caso que nos ocupa se puede concluir que, aunque no puedan extrapolarse los datos obtenidos a medias anuales, si se tiene en cuenta que en estas medidas en condiciones desfavorables las concentraciones de radón interior son muy bajas tanto en invierno como en verano y no existiendo aparentemente ningún otro factor emisor añadido, nada indica que los espacios subterráneos estudiados de la Universidad de Alicante tengan un alto riesgo de albergar una concentración de radón ambiental elevada. Aún así, tal y como se ha comentado, sería necesario una constatación experimental ya que, a pesar de la existencia de rangos de acción en normativas nacionales o internacionales, estos valores nunca deben entenderse como frontera entre niveles "seguros" y "no seguros", siendo siempre deseable minimizar al máximo las concentraciones de radón puesto que, cuanto más altas sean, mayor será el riesgo de desarrollar enfermedades cancerígenas.

\section{AGRADECIMIENTOS}

El presente trabajo ha sido elaborado bajo la concesión a su autora de una Ayuda de Posgrado para la Formación de Profesorado Universitario (FPU) del Programa Nacional de Formación de Recursos Humanos de Investigación, (referencia AP2010-2272) y es parte del Proyecto "Desarrollo y Validación de una Metodología de Clase Mundial para la Evaluación e Intercomparación del Nivel de Sostenibilidad en la Edificación" (referencia IPT-380000-2010-16), del Plan Nacional del I+D+I del Ministerio de Ciencia Innovación, financiado con fondos FEDER.

\section{REFERENCIAS}

(1) WHO. (2000). Air Quality Guidelines for Europe. WHO Regional Publications, European Series, N. ${ }^{\circ}$ 91. Copenhagen.

(2) WHO. (2009). Handbook on indoor radon. A public health perspective.

(3) IAEA. (2003). Radiation protection against radon in workplaces other than mines. Safety Report Series, 33. International Atomic Energy Agency. Viena. 
(4) NTP 440. (2003). Radón en ambientes interiores. Notas Técnicas de Prevención. Instituto Nacional de Seguridad e Higiene en el Trabajo, Madrid.

(5) NTP 533. (2003). El radón y sus efectos sobre la salud. Notas Técnicas de Prevención. Instituto Nacional de Seguridad e Higiene en el Trabajo, Madrid.

(6) NTP 728. (2003). Exposición laboral a radiación natural. Notas Técnicas de Prevención. Instituto Nacional de Seguridad e Higiene en el Trabajo, Madrid.

(7) UE. Directiva 96/29/EURATOM del Consejo de 13 de mayo de 1996 por la que se establecen las normas básicas relativas a la protección sanitaria de los trabajadores y de la población contra los riesgos que resultan de las radiaciones ionizantes. Diario Oficial $\mathrm{n}^{\circ} \mathrm{L} 159$ de 29/06/1996.

(8) UE. Draft European Basic Safety Standards Directive. Versión 24 febrero 2010.

(9) UE. Recomendación 90/143/EURATOM de la Comisión de 21 de febrero de 1990 relativa a la protección de la población contra los peligros de una exposición al radón en el interior de edificios. Diario Oficial n L 080 de 27/03/1990.

(10) Real Decreto 783/2001, de 6 de julio, por el que se aprueba el Reglamento sobre protección sanitaria contra radiaciones ionizantes. BOE n..$^{\circ}$ 178. Madrid, 2001.

(11) Real Decreto 1439/2010, de 5 de noviembre, por el que se modifica el Reglamento sobre protección sanitaria contra radiaciones ionizantes, aprobado por Real Decreto 783/2001, de 6 de julio.

(12) Instrucción IS-33, sobre criterios radiológicos para la protección frente a la exposición a la radiación natural. Consejo de Seguridad Nuclear, 2012.

(13) ICRP 65. (1993). Protection against 222Rn at home and at work, International Commission on Radiological Protection.

(14) Quindós-Poncela, L., Fernández-Navarro, P., Sainz-Fernández, C., Gómez-Arozamena, J., Bordonoba-Perez, M. (2004). Radon exposure in uranium mining industry vs. Exposure in tourist caves. Radiation Protection Dosimetry, 111: 41-44.

(15) Lario, J., Sánchez-Moral, S., Cañaveras, J. C., Cuezva, S., Soler, V. (2005). Radon continuous monitoring in Altamira Cave (northern Spain) to assess user's annual effective dose. Journal of Environmental Radioactivity, 80: 161-174.

(16) Dueñas, C., Fernández, M. C., Cañete, S., Pérez, M., Gordo, E.(2011). Seasonal variations of radon and the radiation exposure levels in Nerja cave, Spain. Radiation Measurements, 46: 1181-1186.

(17) Pinza-Molina, C., Alcaide, J. M., Rodriguez-Bethencourt, R., Hernandez-Armas, J. (1999). Radon exposures in caves of Tenerife (Canary Islands). Radiation Protection Dosimetry, 82: 219-224.

(18) Martín, A., De la Torre, J., Ruano, A. B., Naranjo, F. L. (2012). Radon in workplaces in Extremadura (Spain). Journal of Environmental Radioactivity, 107: 86-91.

(19) Font, LI., Baixeras, C., Moreno, V. (2008). Indoor radon levels in underground workplaces of Catalonia, Spain. Radiation Measurements, 43: S467-S470.

(20) Quindós, L. S., Fernández, P. L., Soto, J. (1995). Study of areas of Spain with high indoor Radon. Radiation Measurements, 24(2): 207-210.

(21) Sainz, C., Dinu, A., Tiberius Dicu, T., Szacsval, K., Cosma, C., Quindós, L. S. (2009). Comparative risk assessment of residential radon exposures in two radon-prone areas, Ştei (Romania) and Torrelodones (Spain). Science of the Total Environment, 407: 44524460.

(22) Barros-Dios, J. M., Ruano-Ravina, A., Gastelu-Iturri, J., Figueiras, A. (2007). Factors underlying residential radon concentration: Results from Galicia, Spain. Environmental Research, 103: 185-190.

(23) Piedecausa-García, B. (2012). La vivienda tradicional excavada: las casas-cueva de Crevillente. Análisis tipológico y medidas de calidad del aire (Tesis doctoral). Universidad de Alicante.

(24) Papachristodoulou, C. A., Patiris, D. L., Ioannides, K. G. (2010). Exposure to indoor radon and natural gamma radiation in public workplaces in north-western Greece. Radiation Measurements, 45: 865-871.

(25) Kotrappa, P., Dempsey, J. C., Stieff, L. R. (1993). Recent advances in electret ion chamber technology for radiation measurements. Radiation Protection Dosimetry, 47: 461464.

(26) Kotrappa, P., Dempsey, J. C., Stieff, L. R., Ramsey, R.W. (1990). A practical electret passive environmental radon monitor for indoor radon measurements. Health Physics, 58: 461-467. 